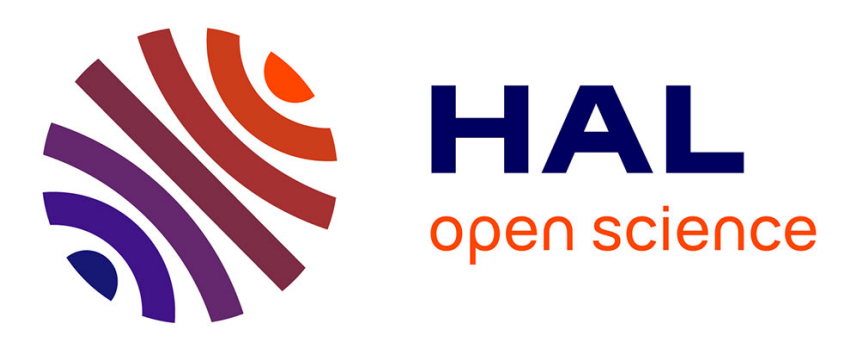

\title{
Quasi-static behavior identification of polyurethane foam using a memory integer model and the difference-forces method
}

\author{
Hamdi Jmal, Raphael Dupuis, Evelyne Aubry
}

\section{- To cite this version:}

Hamdi Jmal, Raphael Dupuis, Evelyne Aubry. Quasi-static behavior identification of polyurethane foam using a memory integer model and the difference-forces method. Journal of Cellular Plastics, 2011, pp.447-465. 10.1177/0021955X11406101 . hal-00851928

\section{HAL Id: hal-00851928 \\ https://hal.science/hal-00851928}

Submitted on 19 Aug 2013

HAL is a multi-disciplinary open access archive for the deposit and dissemination of scientific research documents, whether they are published or not. The documents may come from teaching and research institutions in France or abroad, or from public or private research centers.
L'archive ouverte pluridisciplinaire HAL, est destinée au dépôt et à la diffusion de documents scientifiques de niveau recherche, publiés ou non, émanant des établissements d'enseignement et de recherche français ou étrangers, des laboratoires publics ou privés. 


\title{
Quasi-static behaviour identification of Polyurethane Foam using a memory integer model and the difference-forces method.
}

\author{
H.JMAL, R.DUPUIS* AND E.AUBRY \\ Laboratoire MIPS, Université de Haute Alsace, 12 rue des frères Lumière, 68093 Mulhouse, \\ FRANCE.
}

\begin{abstract}
Flexible polyurethane (PU) foam is widely used in numerous comfort applications such as automotive seat cushions and mattresses. It would be interesting to design a mechanical model which describes the behaviour of this material in a series of test conditions. The present study is devoted to the modelling of the quasi-static behaviour of polyurethane foam using a memory integer model. Polyurethane foam undergoing large compressive deformation exhibits highly nonlinear elasticity and a viscoelastic behaviour. The memory integer model describes the nonlinearity in a polynomial function and the viscoelasticity through a convolution function.

Uni-axial compression tests help to identify the mechanical parameters of the model. The difference between the force responses of foam in load and unload phases constitute the base element of the method used in this paper. Numerous precautions are taken into account to obtain accurate results which verify the thermodynamic conditions. Finally, the reliability as well as the limits of the memory integer model are discussed.
\end{abstract}

KEY WORD: flexible polyurethane foam, quasi-static behaviour, linear viscoelasticity, thermodynamic conditions of model, memory integer model, identification parameters.

\footnotetext{
*Author to whom correspondence should be addressed.

E-mail: raphael.dupuis@uha.fr
} 


\section{Nomenclatures}

\begin{tabular}{|c|c|c|}
\hline Symbols & Units & Definitions \\
\hline$t$ & $(\mathrm{sec})$ & Time \\
\hline$T$ & $(\mathrm{sec})$ & Test period \\
\hline$T_{e c}$ & $(\mathrm{sec})$ & Sampling period \\
\hline$\omega$ & $(\mathrm{Hz})$ & Frequency \\
\hline$x$ & $(\mathrm{~m})$ & Displacement \\
\hline$\left(L_{0} \times l_{0} \times h_{0}\right)$ & $(\mathrm{m} \times \mathrm{m} \times \mathrm{m})$ & Initial dimensions of polyurethane foam samples \\
\hline$\varepsilon$ & $\left(\mathrm{m} \mathrm{m}^{-1}\right)$ & Strain \\
\hline$\dot{\varepsilon}$ & $\left(\sec ^{-1}\right)$ & Strain rate \\
\hline$\varepsilon_{\max }$ & $\left(\mathrm{m} \mathrm{m}^{-1}\right)$ & Maximum strain \\
\hline$\varepsilon_{0}$ & $\left(\mathrm{~m} \mathrm{~m}^{-1}\right)$ & Initial strain \\
\hline$F$ & $(\mathrm{~N})$ & Foam force response \\
\hline$F_{v e}$ & $(\mathrm{~N})$ & Viscoelastic force \\
\hline$F_{e}$ & $(\mathrm{~N})$ & Elastic force (spring force) \\
\hline$K_{i}$ & $\left(\mathrm{~N} \mathrm{~m}^{-\mathrm{i}}\right)$ & Spring stiffness of order $i$ \\
\hline$k_{i}$ & $\left(\mathrm{~N} \mathrm{~m}^{-\mathrm{i}}\right)$ & Global elastic stiffness of order $i$ \\
\hline$K_{v e}$ & $\left(\mathrm{~N} \mathrm{~m}^{-1}\right)$ & Viscoelastic stiffness \\
\hline$\alpha_{l}$ & $\left(\sec ^{-1}\right)$ & Complex number representing the $l^{\text {th }}$ viscoelastic mode \\
\hline $\operatorname{Re}\left(\alpha_{l}\right)$ & $\left(\sec ^{-1}\right)$ & $\begin{array}{l}\text { Real part of } l^{\text {th }} \text { viscoelastic mode representing the } l^{\text {th }} \text { time } \\
\text { relaxation }\end{array}$ \\
\hline $\operatorname{Im}\left(\alpha_{l}\right)$ & $\left(\sec ^{-1}\right)$ & $\begin{array}{l}\text { Imaginary part of } l^{\text {th }} \text { viscoelastic mode representing the } \\
l^{\text {th }} \text { frequency }\end{array}$ \\
\hline$a_{l}$ & $\left(\mathrm{~N} \mathrm{~m}^{-1} \sec ^{-1}\right)$ & Complex number indicating the $l^{\text {th }}$ viscoelastic residue \\
\hline$P$ & & Number of viscoelastic modes \\
\hline$n$ & & $\begin{array}{l}\text { Integer number representing the derivation order associated } \\
\text { to the displacement }\end{array}$ \\
\hline$r$ & & $\begin{array}{l}\text { Integer number representing the derivation order associated } \\
\text { to the viscoelastic force }\end{array}$ \\
\hline$\left(c_{i}\right)_{i=0 . n}$ & & Real coefficients associated to the displacement \\
\hline$\left(b_{i}\right)_{i=0 . . r}$ & & Real coefficients associated to the viscoelastic force \\
\hline $\begin{array}{l}M \\
N\end{array}$ & & $\begin{array}{l}\text { Order of elastic polynomial } \\
\text { number of spicemens by test }\end{array}$ \\
\hline$N_{c y c}$ & & Number of cycles in quasi-static tests \\
\hline
\end{tabular}




\section{INTRODUCTION}

Polyurethane foam is a cellular material characterized by an interesting spectrum of mechanical properties [1] such as: low density (generally less than $80 \mathrm{~kg} \mathrm{~m}^{-3}$ for flexible foam), the ability to absorb the strain energy and low stiffness. This spectrum makes foam a reliable mechanical shock absorber and a major engineering material widely used in numerous comfort applications such as automotive seat cushions and mattresses.

To characterize foams, several standards, such as the American standard D3574-95 of the American Society for Testing Material [2], specify the methods and tests for the assessment of foam properties. Among the tests, there is the indentation force deflection test, the compression force deflection test, the ball rebound test, and dynamic tests such as transmissivity and impact tests. A number of studies have considered foam behaviour in different test conditions. In mechanics, there are four types of studies: static behaviour [3, 4] , quasi-static behaviour [5-9], dynamic behaviour [9-14] and fatigue behaviour [15, 16]. All these studies show that foam cannot be considered as a purely elastic material or as a purely viscous material. They confirm that polyurethane foam has a viscoelastic behaviour. In the case of the static study, the creep and relaxation tests show that creep function is neither time independent nor time proportional. The hysteresis cycle observed on the response displacement force of polyurethane foam undergoing large compressive loading and unloading deformation highlights the viscoelastic behaviour. In the case of dynamic design, the force-displacement phase difference is between $\frac{\pi}{2}$ and 0 . This illustrates another manifestation of the foam viscoelastic behaviour.

Two types of analyses help to predict the mechanical response of polyurethane foams: microscopic analysis and macroscopic analysis.

The micro-mechanical models consider the fundamental components of foam such as shape cells, facets and beam cells (e.g. cubic and tetrahedral cells are considered respectively by Gibson L.J et al. [1] and Warren W.E et al. [17, 18] ). In the first step, the mechanical parameters of only one cell are determined in a simple analytic study. The mechanical response is then predicted using the finite elements method or finite volumes method or else the Vornoi technique which supposes a random distribution of different shape cells [19, 20]. Finally, the model is adjusted by comparing the simulated response and experimental response. The microscopic analysis develops relationships between the geometric properties of cells and the elastic properties of foam. It shows that foam undergoing large deformation exhibits a nonlinear elastic behaviour. However, this analysis is cannot predict dynamic properties.

Contrary to the microscopic analysis, the macroscopic analysis considers the overall response of a foam sample in order to estimate the macro-mechanical material parameters such as the global Young modulus, quasi-static and dynamic stiffness, the damping coefficient and viscoelastic compounds. In the framework of this analysis, there are two types of models: the energetic and memory models. 
The analytic energetic models determines the strain energy function from the Cauchy Green left tensor. They can depend on one material parameter (e.g. Yeoh model [21], Arruda-Boyce model [22], and Neo-Hookean model [23]) or on two material parameters [6] (e.g. MooneyRivlin model and Van der Waals model). The Ogden model [24, 25] is a more general energetic model which is supposed to be composed of multiple Green Lagrange generalized models. These energetic models confirm the nonlinear elastic behaviour of foam undergoing large deformation.

As regards specific foam macroscopic memory models, there are two types of models: the integer memory model $[9,11-13]$ and the fractional memory model $[5,10]$. They illustrate the historical effect on the behaviour of foam. Indeed, in the case of the quasi-static or dynamic compression tests, the foam response, in current calculus instant, is influenced by all the previous response values. The memory models describe the nonlinearity of elastic behaviour through a polynomial function and the viscoelasticity in a convolution function which is derived from an ordinary derivation equation in the case of the integer model or from a fractional derivation equation in the case of the fractional model. The integer memory model will be presented in this paper.

To identify the integer model parameters, Singh R. considers a dynamic compression test. In his first work, he models polyurethane foam using linear integer model (linear elastic behaviour with a convolution function for viscoelastic behaviour) and develops an analytical identification process in the case of low amplitude impact tests [11]. In his second work , Singh R. also considers a linear integer model and estimates the dynamic properties of foam with a Prony series [12]. In a final work, Singh R. identifies the nonlinear integer model parameters using the balance harmonics when the input test (displacement) is sinusoidal [12]. Recently, Ippili R.K. has considered a quasi-static compression test [9]. In his work, the average curve between load and unload phases constitutes the initial elastic polynomial for his method. It will then be adjusted in order to obtain the best correspondence between the experimental and model force-displacement curves. The viscoelastic parameters are obtained using a linear regression estimation method (e.g. ARMA method). This method is called average force method.

To identify the fractional model parameters, Deng R. has developed a method based on the elastic force symmetry between the load and unload quasi-static compression phases [5]. The experimental curve, representing the difference forces between the load and unload phases, is then determined and the viscoelastic parameters are identified using minimizing methods of the average least square error between the latter curve and the same model curve. If the difference-forces function is not convex, the minimizing process can converge to local minimum and the results of identification are inaccurate.

The average force is just an initial supposition of elastic response with no fundamental physical bases. However, the symmetry of elastic response hypothesis is assumed to be logical and physical.

In this paper, an integer memory model is considered to study the behaviour of polyurethane foam undergoing large quasi-static compressive deformation. The model parameters are identified by using difference between load and unload forces method. An approach is developed to insure large probability to find accurate results. 


\section{EXPERIMENTS}

\section{Polyurethane foam samples}

Polyurethane foam, designated by foam Type $\mathrm{A}$ in the tests, has the same characteristics as seat car foam. The properties of foam Type A are summarized in Table 1.

To ensure reliable and generalizable results, 81 specimens of foam Type A have been used (Table 2: $N=45$ for the test $\mathrm{n}^{\circ} 1, N=17$ for the test $\mathrm{n}^{\circ}$, and $N=19$ for the test $\mathrm{n}^{\circ}$ ). All specimens have the same mechanical and environmental histories: they are virgin specimens and have been obtained by cutting mattresses of dimension $2000 \mathrm{~mm}$ x $1200 \mathrm{~mm}$ x $75 \mathrm{~mm}$ into cubic samples. Every specimen has been compressed only one time.

The minimum numbers of specimens by test are calculated to insure a good accuracy for the statistic results. The procedure is more detailed in the section 'VALIDATION RESULTS AND DISCUSSION'.

Table 1. Chemical and morphological foam Type A characteristics.

\begin{tabular}{ll}
\hline Material & Characteristics \\
\hline Designation & PUR : Type A \\
Foam type & Flexible polyurethane foam \\
Isocynate & Toluene diisocynate TDI \\
Polyol & Polyether \\
Expansion agent & $\mathrm{CO}_{2}$ \\
Fabrication process & Free rise \\
Density & $28 \mathrm{~kg} \mathrm{~m}^{-3}$ \\
Porosity & $800 \mu \mathrm{m}$ \\
Samples shape & cubic \\
Dimensions $\left(L_{0} \times l_{0} \times h_{0}\right)$ & $0.075 \mathrm{~m} \times 0.075 \mathrm{~m} \times 0.075 \mathrm{~m}$ \\
Cells type & open \\
\hline
\end{tabular}

\section{Quasi-static compression test and experimental device}

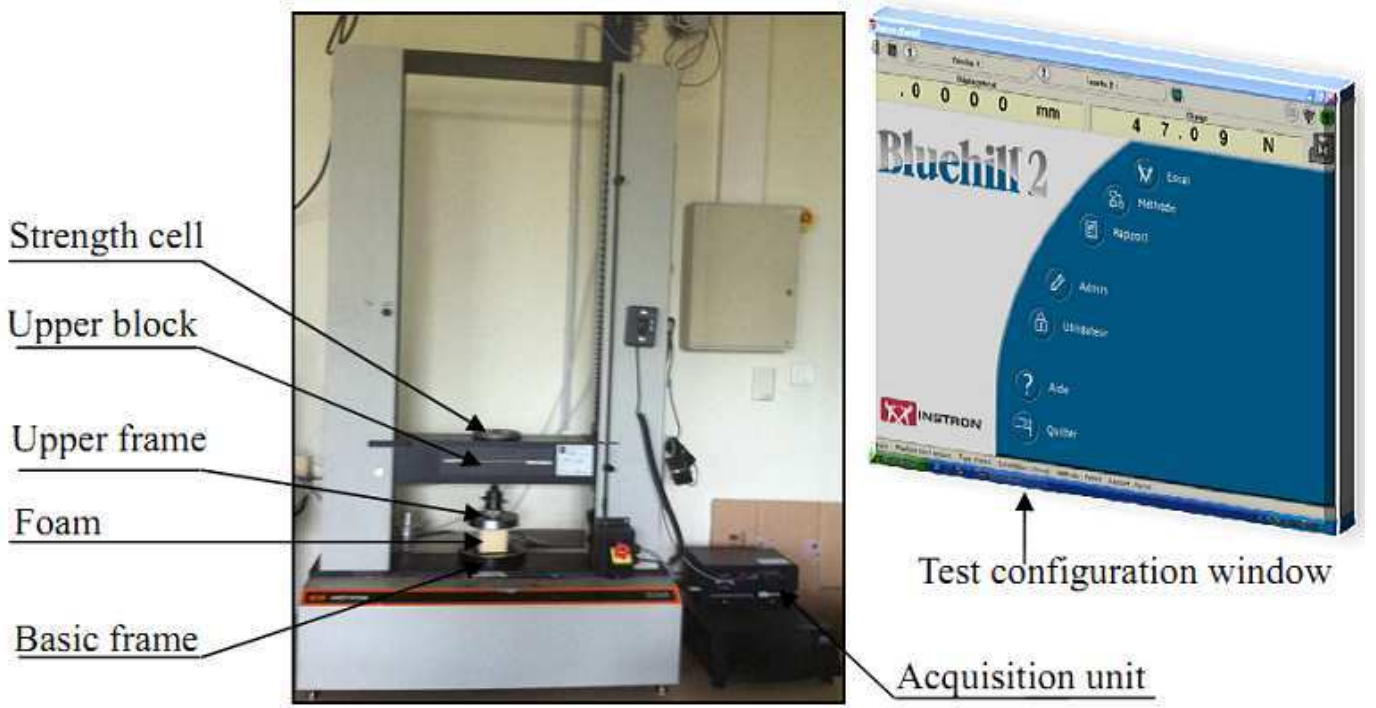

Figure 1. Quasi-static compression test device 
The quasi-static compression device 'Instron 33R4240' (tension-compression machine) includes a basis frame and an upper block which moves vertically. The quasi-static test consists in sandwiching the foam sample between the upper frame and the basis machine up to final compression level (load phase) and in unloading progressively up to initial compression level (unload phase, second step). To minimize the noise contribution, the maximum experimental response force of foam must be slightly less than the load cell maximum capacity. Displacement and force sensors are already integrated in the machine. The number of cycles, the test conditions (maximum compression level, strain rate, etc.), the sampling period and mechanical properties to extract are defined through the 'Blue Hill' test configuration Window.

Foam Type $\mathrm{A}$ is initially between the upper frame and the basis machine. The test begins when the upper frame affects the foam. The quasi-static compression tests have two phases of load and unload. In the load phase, the upper block moves vertically compressing the foam to the final level defined previously. In the second phase, the upper block changes direction to return to its starting position. The strain rate is considered constant in the two phases.

The conditions tests are grouped in following table:

Table 2. Quasi-static compression test conditions

\begin{tabular}{l|lllllll}
\hline & $N_{c y c}$ & $\dot{\varepsilon}\left(\mathrm{sec}^{-1}\right)$ & $\varepsilon_{0}(\%)$ & $\varepsilon_{\max }(\%)$ & $T(\mathrm{sec})$ & $T_{\text {ech }}(\mathrm{sec})$ & $N$ \\
\hline Test $\mathrm{n}^{\circ} 1$ & 1 & $1.0610^{-2}$ & 0 & 80 & 150 & 0.0625 & 45 \\
Test $\mathrm{n}^{\circ} 2$ & 1 & $5.3310^{-3}$ & 0 & 80 & 300 & 0.125 & 17 \\
Test $\mathrm{n}^{\circ} 3$ & 1 & $6.6610^{-4}$ & 0 & 80 & 2400 & 2 & 19 \\
\hline
\end{tabular}
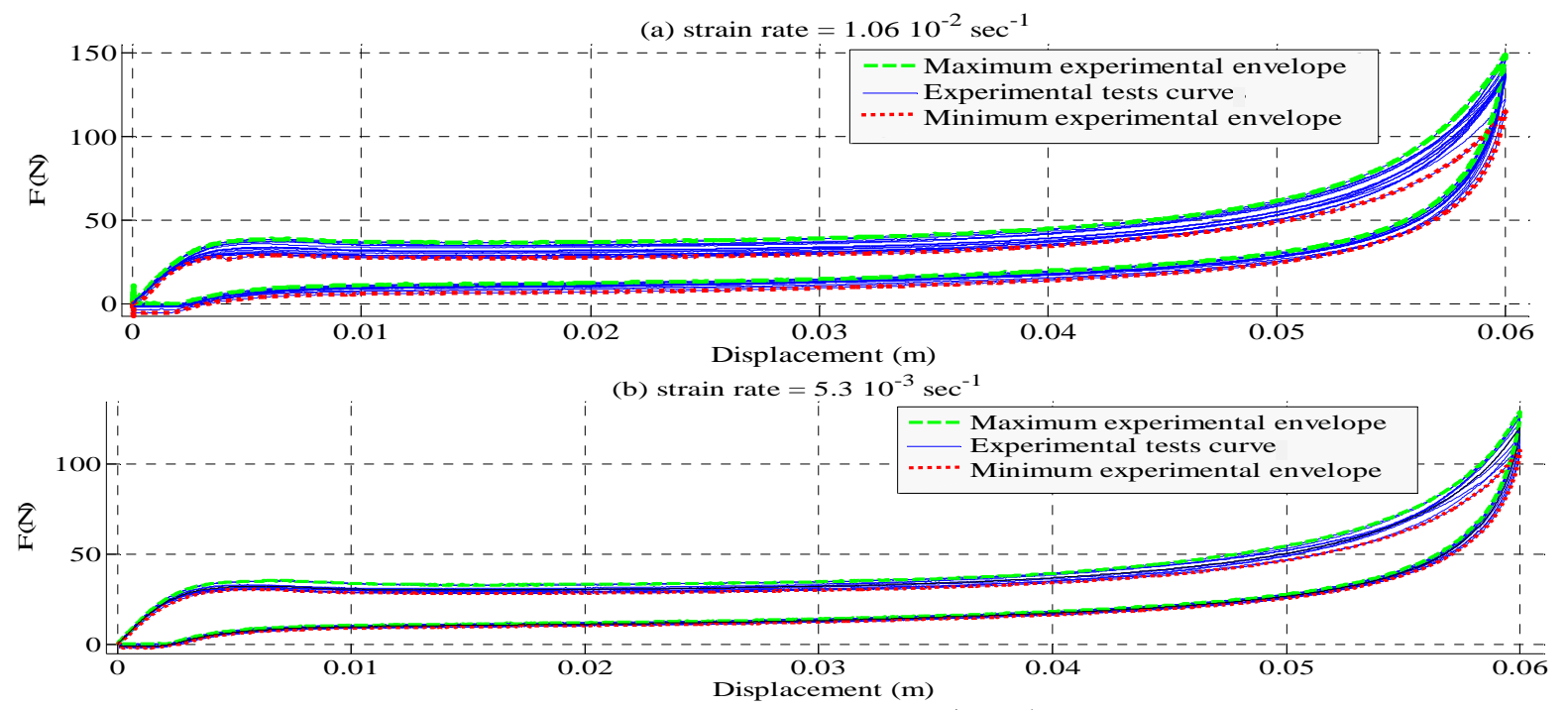

(c) strain rate $=6.6610^{-4} \mathrm{sec}^{-1}$

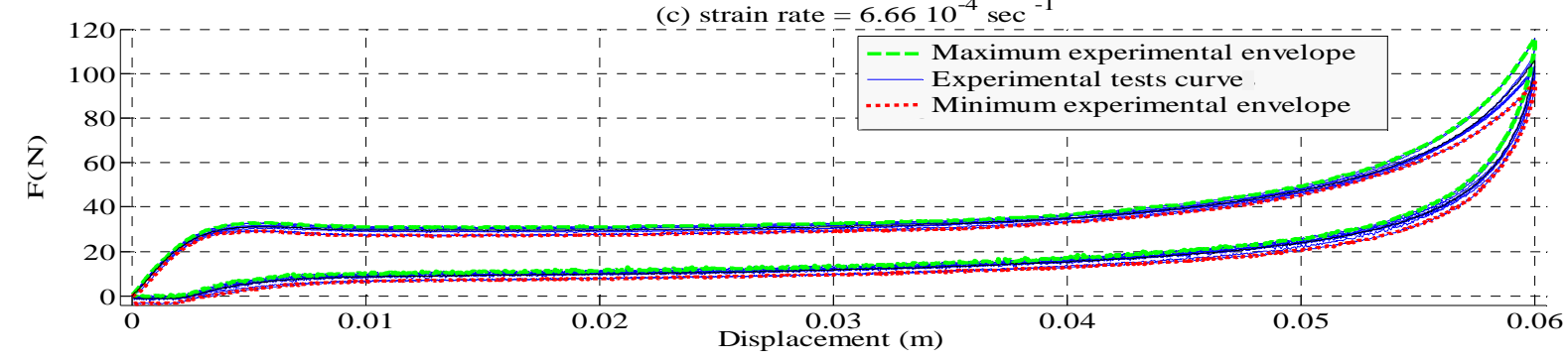

Figure 2. Experimental force-displacement curves 
The experimental force-displacement response of foam Type A, for all tests, is presented in Figure 2. These tests allow the extraction of the minimum and maximum experimental envelopes. The experimental results of the tests show a hysteresis foam response. It is also observed that the dispersion during the load phase seems more important than the dispersion during the unload phase.

\section{MODELLING}

Polyurethane foam undergoing large compressive deformation is assumed to be homogeneous, isotropic with constant cross-section [6]. It exhibits highly nonlinear elasticity and viscoelastic behaviour.

\section{Elastic behaviour}

The elastic foam component is typically modelled by a nonlinear spring described by a power function. In this paper, it is modelled by a polynomial function:

$$
F_{e}(t)=\sum_{i=1}^{M} K_{i}(x(t))^{i}
$$

\section{Viscoelastic behaviour}

In the literature, numerous elementary macroscopic models are used to describe the viscoelastic behaviour, such as the Maxwell model (spring and dash pot in series), the Kelvin Voigt model (spring and dash pot in parallel), and the Zener model (Maxwell model in series with a spring). Assuming a random combination of elementary models in series and parallel the viscoelastic behaviour is given by:

$$
b_{0} F_{v e}(t)+b_{1} \frac{d^{1} F_{v e}(t)}{d t}+\ldots+b_{r} \frac{d^{r} F_{v e}(t)}{d t^{r}}=c_{0} x(t)+c_{1} \frac{d^{1} x(t)}{d t}+\ldots+c_{n} \frac{d^{n} x(t)}{d t^{n}}
$$

The integer model of viscoelasticity (equation 3.) is obtained when $r>n, r>2$ and the all poles of the impulsion response are distinct. In this study, $P$ is considered equal to two.

$$
F_{v e}(t)=\int_{0}^{t} \sum_{l=1}^{P} a_{l} e^{-\alpha_{l}(t-\tau)} x(\tau) d \tau
$$

If $r=n, r>2$ and $c_{0}=0$, the viscoelastic force is generally composed of purely elastic component and memory terms:

$$
F_{v e}(t)=K_{v e} x(t)+\int_{0}^{t} \sum_{l=1}^{P} a_{l} e^{-\alpha_{l}(t-\tau)} x(\tau) d \tau
$$

\section{Non linear integer model}

The convolution product takes account of the memory criterion of foam. The polynomial function indicates the nonlinear behaviour. For these reasons, the global model of foam undergoing large compressive deformation is called nonlinear memory integer model:

$$
F(t)=F_{v e}(t)+F_{e}(t)=\int_{0}^{t} \sum_{l=1}^{P} a_{l} e^{-\alpha_{l}(t-\tau)} x(\tau) d \tau+\sum_{i=0}^{M} k_{i}(x(t))^{i}
$$




\section{Thermodynamic conditions}

The thermodynamic conditions are the causality and stability conditions of the model. They are determined from the Fourier transform $G(j \omega)$ (equation 5.) of the pulse model response, neglecting nonlinear components.

$$
G(j \omega)=k_{1}+\sum_{l=1}^{P} \frac{a_{l}}{j \omega-\alpha_{l}}
$$

To ensure the stability and causality of the model, it is necessary to verify the following conditions:

$$
\begin{cases}\operatorname{Re} a l(G(j \omega)) \geq 0 & \forall \omega \geq 0 \\ \operatorname{Im}(G(j \omega)) \geq 0 & \forall \omega \geq 0\end{cases}
$$

Considering the two cases of $\omega \mapsto 0$ and $\omega \mapsto+\infty$, the thermodynamic conditions of the integer model are grouped in following table:

Table 3. Thermodynamic conditions of the macroscopic integer model

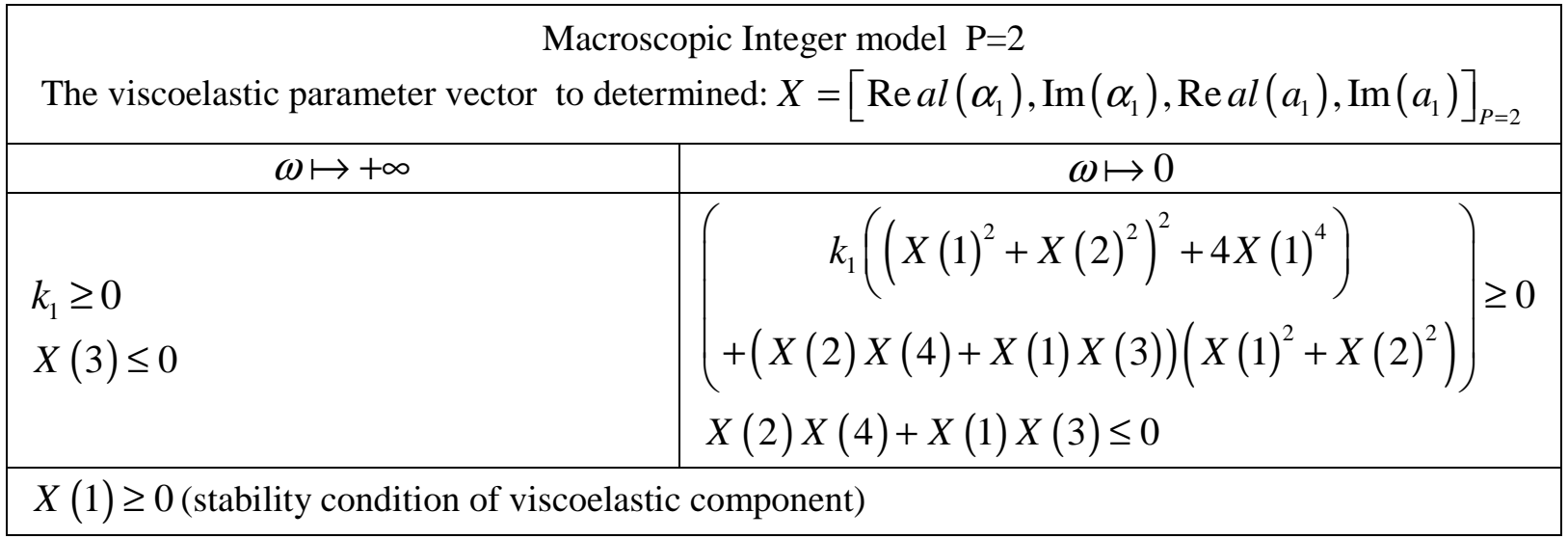

\section{IDENTIFICATION METHOD}

\section{Difference-forces method}

In the case of the quasi-static compression test, the strain rates in load and unload are considered equal. So, the displacement curve $x(t)$ is represented as an isosceles triangle (Figure 3.). The times $\left(t_{1}, t_{2}\right)$ correspond to the same displacement $x_{0}$ respectively in the load and unload phases. They are related by the following expression:

$$
t_{2}=T-t_{1}
$$

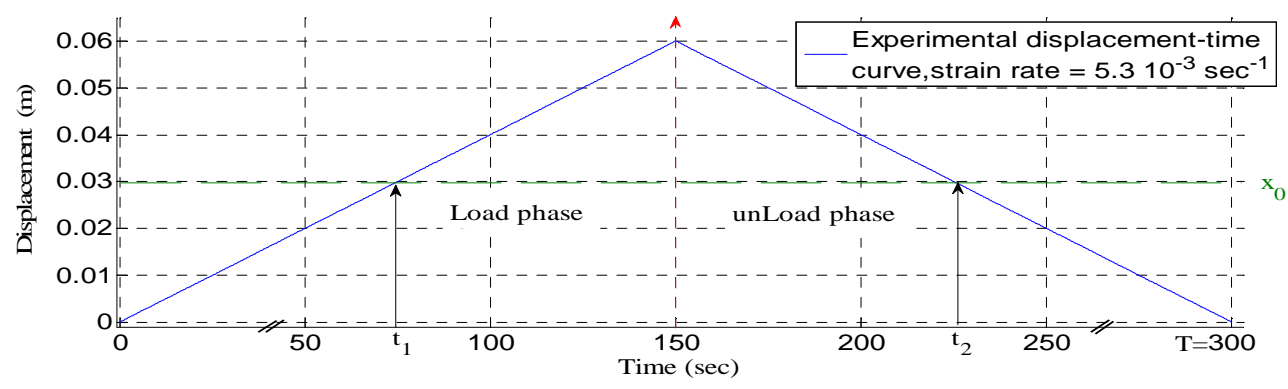

Figure 3. Experimental displacement-time curve 
The difference-forces method is based on the symmetry of the elastic force between the load and unload phases. So only the viscoelastic parameters are present in the analytical expression of the difference forces between load and unload:

$$
\Delta F_{t h}\left(x_{0}\right)=\int_{0}^{t_{1}} \sum_{i=1}^{P} a_{i} e^{-\alpha_{i}\left(t_{1}-\tau\right)} x(\tau) d \tau-\int_{0}^{t_{2}} \sum_{i=1}^{P} a_{i} e^{-\alpha_{i}\left(t_{2}-\tau\right)} x(\tau) d \tau
$$

Using the previous equations 8 and 9, the load time projection of the difference forces is determined:

$$
\Delta F_{t h}\left(t_{1}\right)=\sum_{i=1}^{P} \frac{l_{0} \dot{\varepsilon}}{\alpha_{i}^{2}} a_{i}\left(e^{-\alpha_{i} t_{1}}+2 e^{\alpha_{i}\left(t_{1}-\frac{T}{2}\right)}-e^{-\alpha_{i}\left(T-t_{1}\right)}-2\right)
$$

The difference-forces method consists in establishing, through an optimization method, the viscoelastic parameters which allows help to minimize the least mean square error between the analytical and experimental difference forces (Figure 4.). In the second step, the reconstruction of the viscoelastic force in the load and unload phases allows the determination of the elastic force. Then, the symmetry condition of the elastic force must be verified and the elastic polynomial coefficients are identified using MATLAB.

To improve the accuracy of the viscoelastic model parameters while minimizing calculation time, the real form of equation 10 is worth developing:

$$
\begin{aligned}
& \Delta F_{t h}\left(t_{1}\right)=\frac{2 l_{0} \dot{\varepsilon}}{\left(X(1)^{2}+X(2)^{2}\right)^{2}}\left(\begin{array}{c}
-2 A \\
+\left(A \cos \left(X(2) t_{1}\right)+B \sin \left(X(2) t_{1}\right)\right) e^{-X(1) t_{1}} \\
-\left(A \cos \left(X(2)\left(t_{1}-\frac{T_{1}}{2}\right)\right)-B \sin \left(X(2)\left(t_{1}-\frac{T_{1}}{2}\right)\right)\right) e^{-X(1)\left(t_{1}-\frac{T_{1}}{2}\right)} \\
\left.-\left(X(2)\left(t_{1}-T_{1}\right)\right)-B \sin \left(X(2)\left(t_{1}-T_{1}\right)\right)\right) e^{-X(1)\left(t_{1}-T_{1}\right)}
\end{array}\right) \\
& \text { with }\left\{\begin{array}{l}
X=\left[\operatorname{Re} a l\left(\alpha_{1}\right), \operatorname{Im}\left(\alpha_{1}\right), \operatorname{Re} a l\left(a_{1}\right), \operatorname{Im}\left(a_{1}\right)\right]_{P=2} \\
A=X(3)\left(X(1)^{2}+X(2)^{2}\right)+2 X(1) X(2) X(4) \\
B=X(4)\left(X(1)^{2}+X(2)^{2}\right)-2 X(1) X(2) X(3)
\end{array}\right. \\
& \Delta \mathrm{F}_{\mathrm{exp}}(\mathrm{N}){ }_{10}
\end{aligned}
$$

Figure 4. The projection of the difference forces between load and unload phases on load time The algorithm of this method is summarized in Figure 5. 


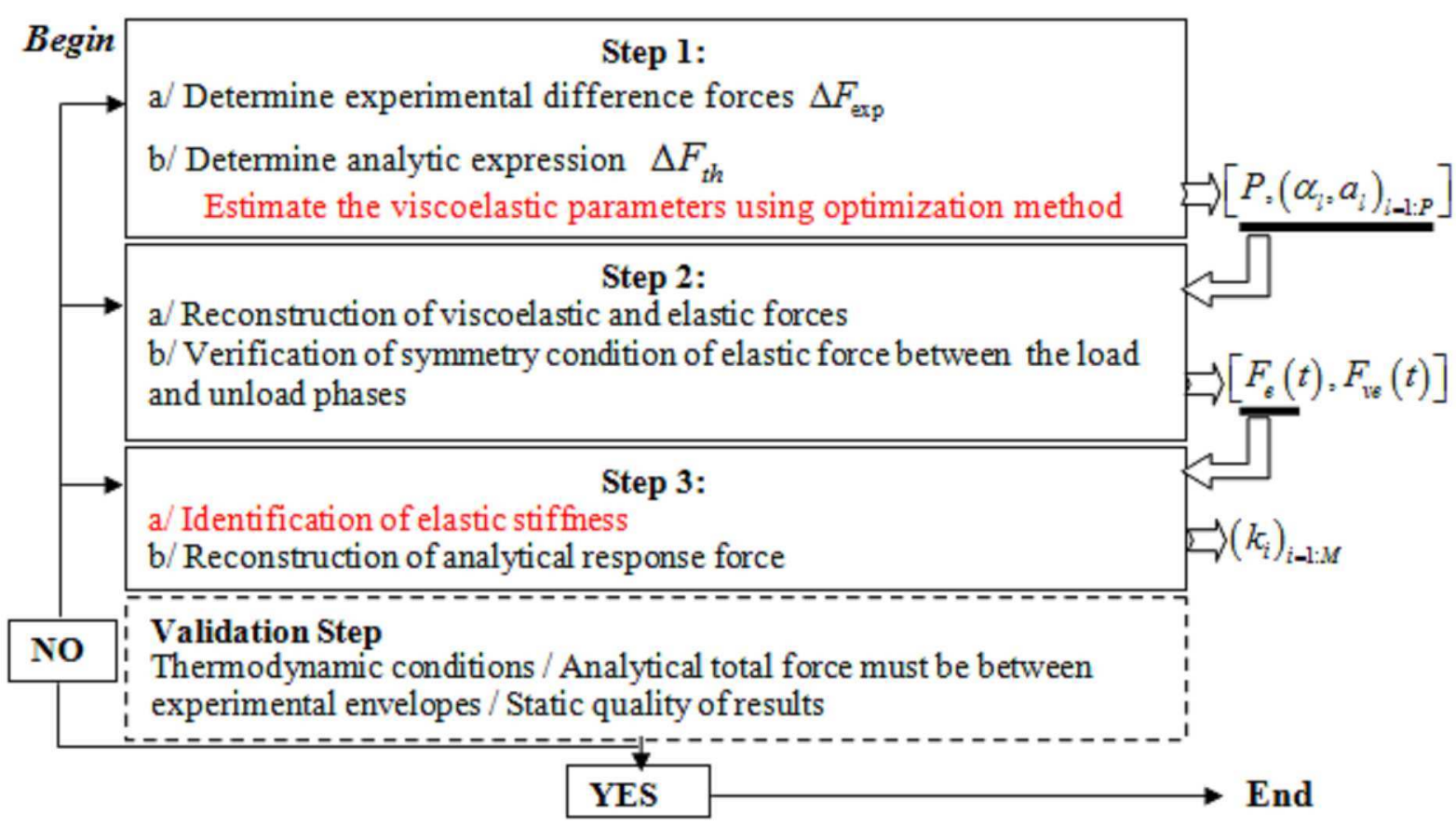

Figure 5. Algorithm of the difference-forces method.

\section{Optimization approach}

Optimization methods are the basic tools for viscoelastic parameter identification. There are two types of optimization methods - deterministic and random methods.

Deterministic methods (e.g. Gauss-Seidel, Trust region reflective, Levenberg-Marquardt and Gradient methods) are effective when the objective function (function to optimize) changes rapidly and when it has a known prior form so that it is possible to choose an initialization near the global minimum. However, in the general case, these methods are not able to find the global minimum.

Random methods (e.g. Monte Carlo, Nelder-Mead algorithm and genetic algorithm) are reliable in the case of functions which cannot be differentiated and in the case of fractal and noisy functions. In addition, these methods offer great probability to find the global minimum when the objective function has numerous local minima. However, they require excessive calculation time compared with deterministic methods.

In order to exploit the advantages of deterministic methods while ensuring high probability to find the global minimum, an approach in three steps is considered. It consists in using both the genetic algorithm ('gatool' command of MATLAB) and the Trust region reflective method ('Isqnonlin' command). The first step gives a great chance to find the initialization for the lsqnonlin solver near the global optimum using the genetic algorithm with gross stopping criteria and without boundary constraints. In the second step, the search for the best combination of viscoelastic parameters which minimizes the least mean square error between the analytical and experimental difference forces is finalized using the lsqnonlin MATLAB solver with tight stopping criteria. Finally, the solution found in the second step is injected into a random initial population of genetic algorithm to verify that it is effectively the global minimum. The stopping criterion in this third step is the generation number fixed at 100 . For the steps two and three, the thermodynamic conditions are used as boundary constraints. 


\section{VALIDATION RESULTS AND DISCUSSION}

The viscoelastic parameters found by Ippili R.K. [9] in the case of a quasi-static study depends on the test conditions (strain rate, final compression level, test period ). In this study, the idea is to replace the viscoelastic parameters by dimensionless parameters (Table 4) in order to determine any possible relations between viscoelastic parameters and test conditions.

Table 4. Dimensionless viscoelastic parameters

\begin{tabular}{lll}
\hline \multicolumn{2}{l}{ Dimensional viscoelastic parameters } & Dimensionless parameters \\
\hline$X(1)=\operatorname{Re}\left(\alpha_{1}\right)$ & $\left(\sec ^{-1}\right)$ & $Y(1)=X(1) T$ \\
$X(2)=\operatorname{Im}\left(\alpha_{1}\right)$ & $\left(\sec ^{-1}\right)$ & $Y(2)=X(2) T$ \\
$X(3)=\operatorname{Re}\left(a_{1}\right)$ & $\left(\mathrm{N} \mathrm{m}^{-1} \sec ^{-1}\right)$ & $Y(3)=\frac{X(3)}{F_{\text {max }}} l_{0} \varepsilon_{\max } T$ \\
$X(4)=\operatorname{Im}\left(a_{1}\right)$ & $\left(\mathrm{N} \mathrm{m}^{-1} \sec ^{-1}\right)$ & $\frac{X(4)}{F_{\text {max }}} l_{0} \varepsilon_{\max } T$ \\
\hline & $F_{\text {max }}=\max (F)$ is determined from tests
\end{tabular}

The identification results are grouped in the following table:

Table 5. Parameter identification results

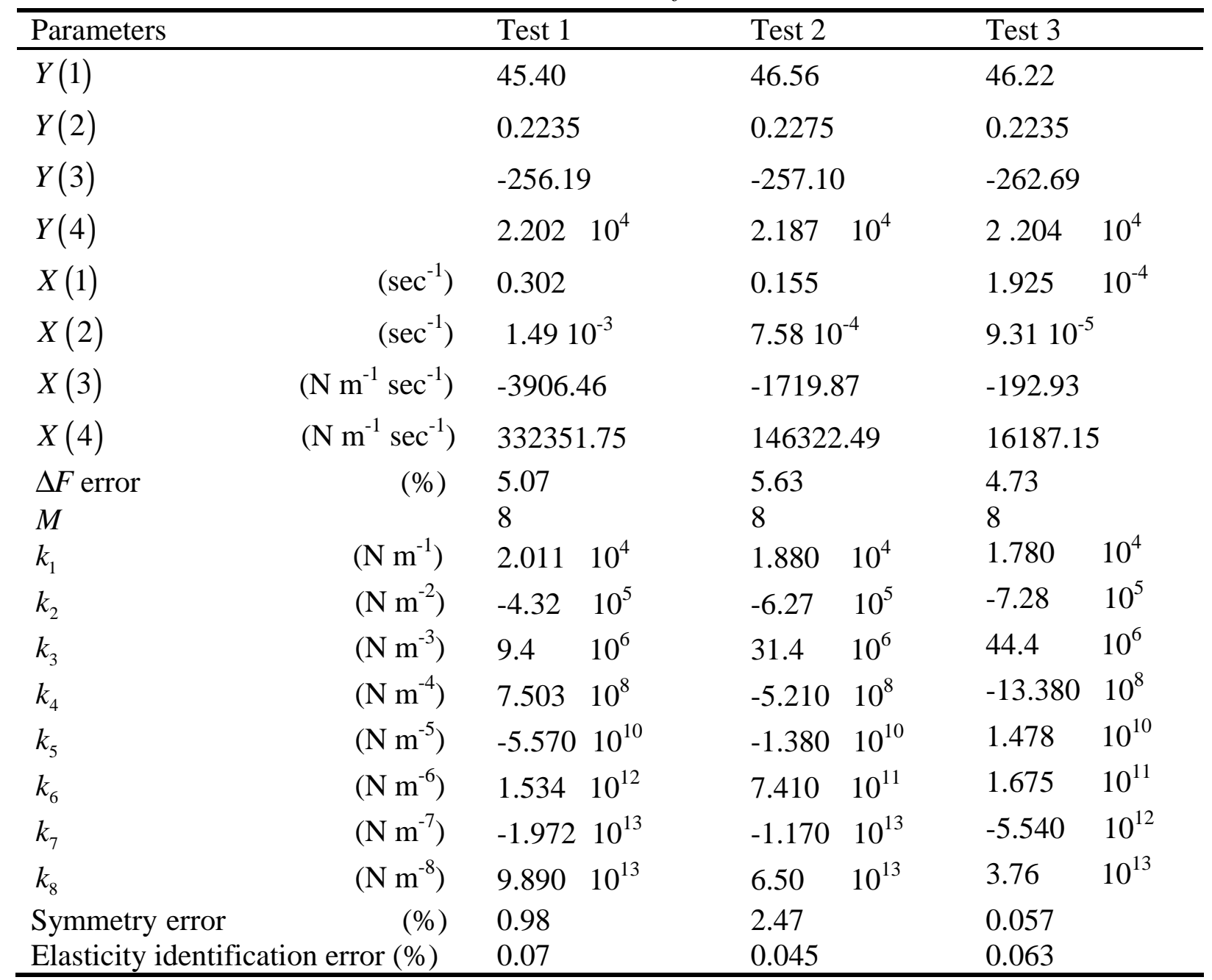


To validate these results, it is necessary to examine five criteria: the thermodynamic conditions, statistic quality, accurate $\Delta F$ estimation, elastic symmetry hypothesis and the model force response between the experimental envelopes.

The parameter results verify the thermodynamic conditions presented in table 3 .

They are the mean of $N$ test samples (Table 2.) which is determined to ensure the statistical quality of all identified parameters for each strain rate (second criteria). This quality is reviewed through the set at a 95\% confidence level and the statistical error limit "SLE" which must not exceed $10 \%$. The minimum number of test samples for each strain rate is calculated using the following equation:

$$
N=\max _{l}\left(1+\operatorname{ceil}\left(\left(\frac{100}{S L E} \frac{u_{l} \hat{\sigma}_{l}}{\hat{m}_{l}}\right)^{2}\right)\right)
$$

$\left(\hat{m}_{l}, \hat{\sigma}_{l}\right)$ are the estimated average and standard deviation values corresponding to $l^{\text {th }}$ parameter. They are calculated from the identification results of the preliminary test battery (15 test samples). $u_{l}$ is a coefficient determined from a probability table suitable for the estimated probability law of $l^{\text {th }}$ parameter. In this paper, the Student law is assumed for all parameters.

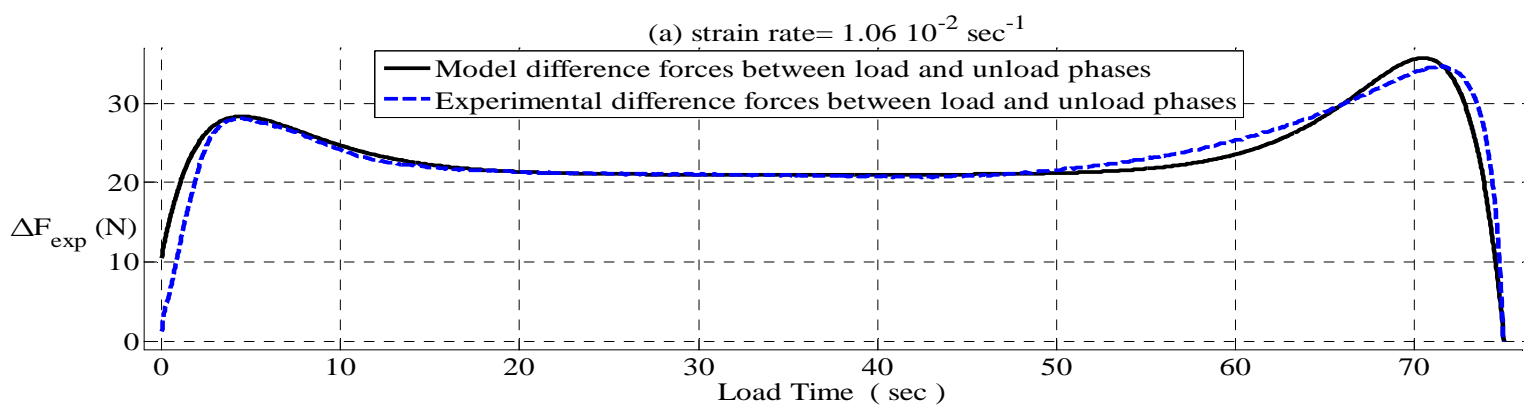

(b) strain rate $=5.310^{-3} \mathrm{sec}^{-1}$

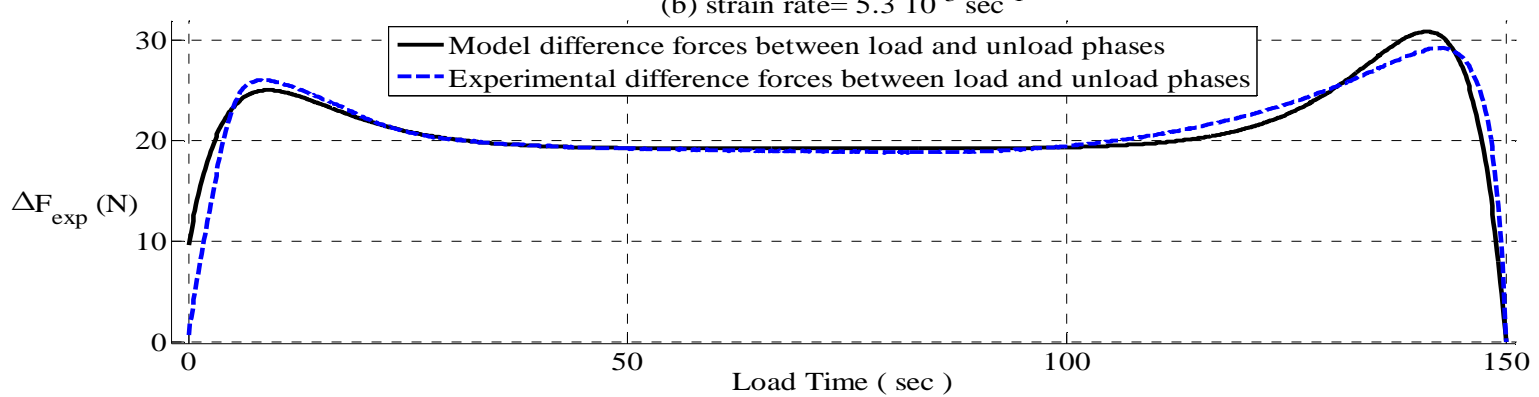

(c) strain rate $=6.6610^{-4} \mathrm{sec}^{-1}$

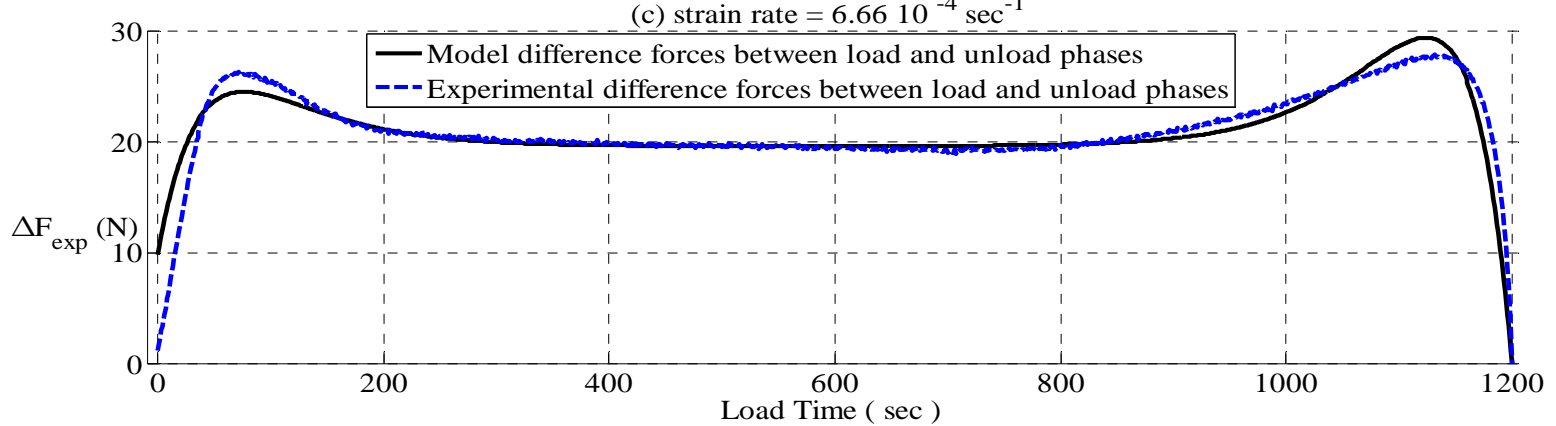

Figure 6. Comparison between model and experimental difference-forces

The third criterion is the comparison of model and experimental difference-forces between load and unload phases, as shown in figure 6. The maximum relative error between the 
experimental and analytical curves is in the order of 5\%. It is also shown in figure 6 that the initial part of the analytical curve has shifted compared with the experimental curve. This is explained through the interpretation of figure 7. In this figure, it is shown that the model reconstructs the foam force response in good similarity with the experimental force response. But at the end of the test, the model and experimental responses are not the same and the difference is very important. In fact, a residual stress pushes foam to return into a final position, different from the initial one before the test. This is a result of the viscoelasticity behaviour. So, the contact between the upper frame and the foam is lost and the experimental force response is inaccurate.

Figure 7. shows that the foam model anticipates the force response between the minimum and maximum experimental envelopes. So the fourth criterion is verified.
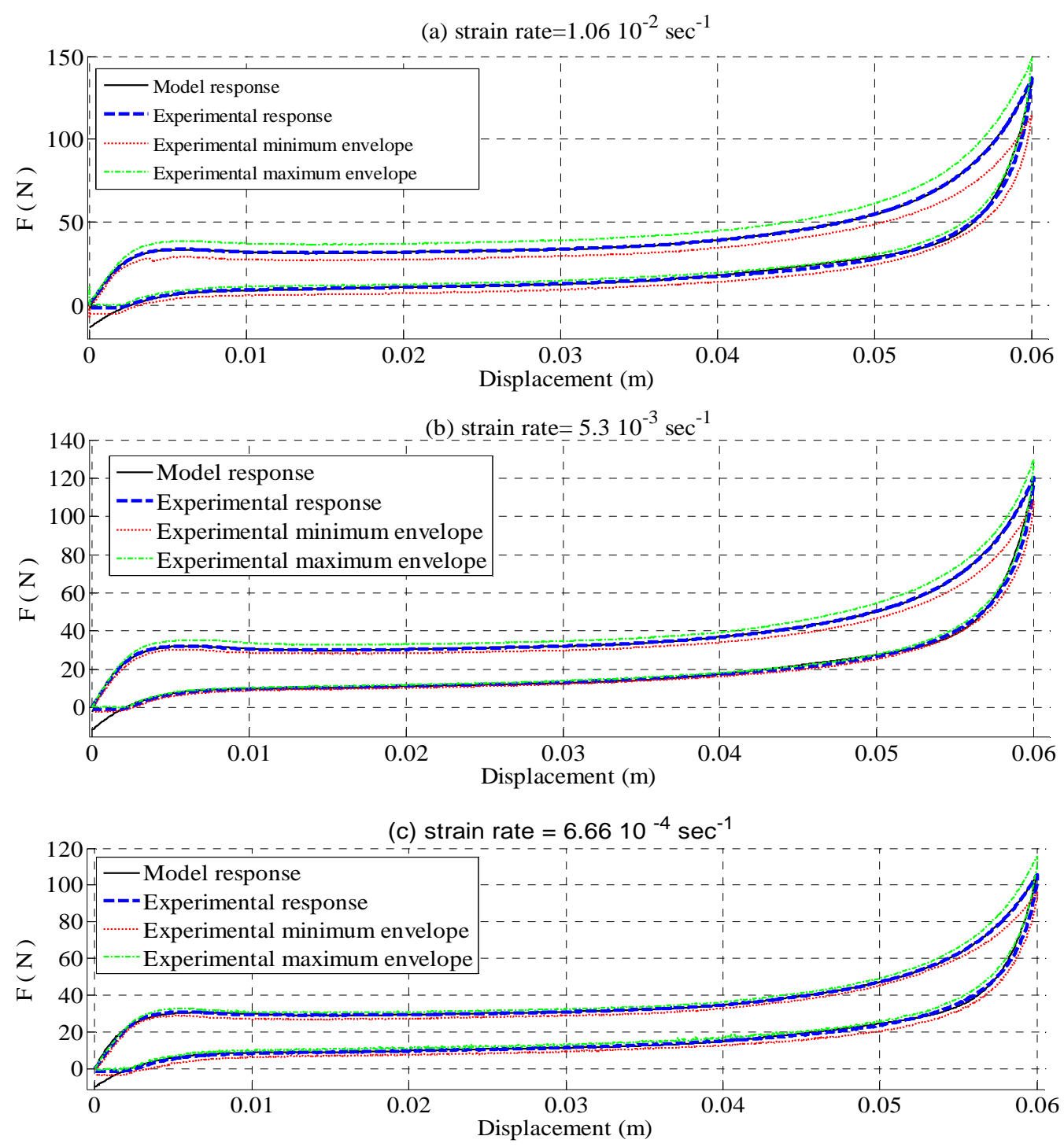

Figure 7. Reconstruction of the force response of foam

The symmetry of the elastic force between the load and unload phases is the basis assumption of difference-forces method. Therefore, the symmetry condition must be verified to validate the identification parameters. The reconstruction of the global elastic response, presented in figure 8 , shows that the model results verify the elastic symmetry. This is the fifth and last criterion. 
(a) strain rate $=1.0610^{-2} \mathrm{sec}^{-1}$
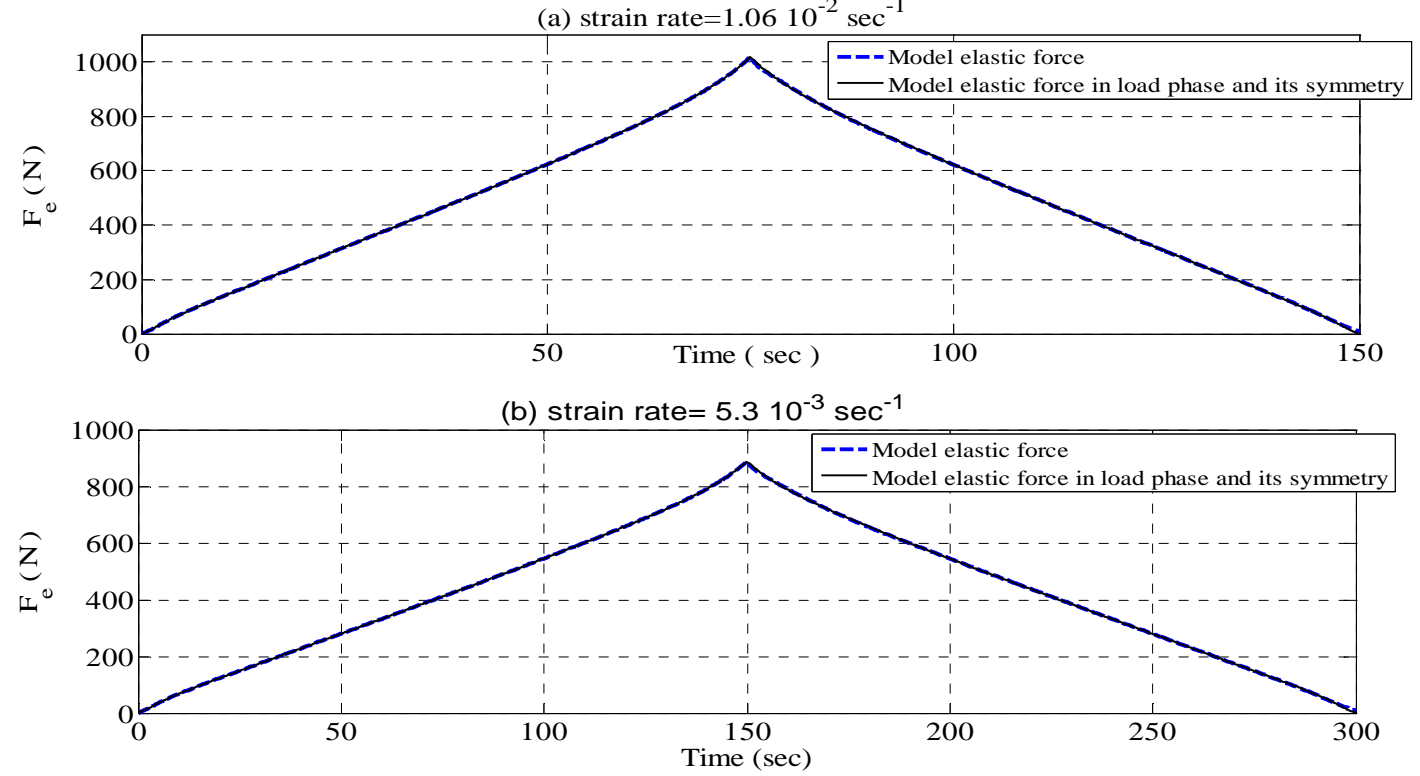

(c) strain rate $=6.6610^{-4} \mathrm{sec}^{-1}$

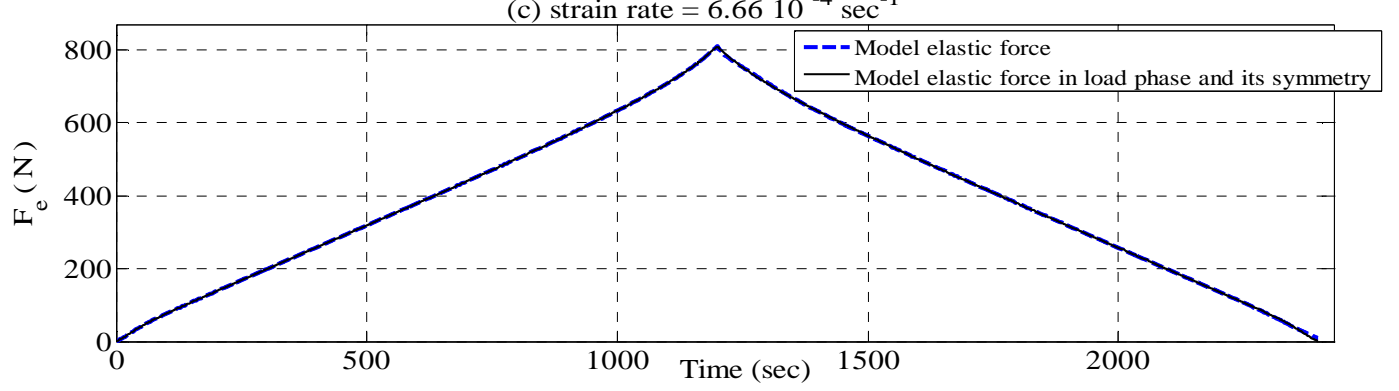

Figure 8. Verification of the elastic force symmetry

It has been shown that the viscoelastic dimensionless parameters are invariant for all strain rates. So it is possible to characterize the viscoelastic behaviour of polyurethane with these parameters independently of test conditions. The use of dimensionless parameters makes optimization algorithms faster. Moreover, it improve the conditioning matrix used in the estimation method.

However, the order value of the global elastic response and of the viscoelastic response (Figure 9.) is very important. The global elastic polynomial is almost linear (figure 8). This contradicts the studies presented in the introduction and the first assumption: foam under large deformation exhibits a nonlinear elastic behaviour. The linearity is due to the dominance of the global stiffness response of the first order $\left(k_{1} x \gg \sum_{i=2}^{M} k_{i} x^{i}\right)$. This stiffness $k_{1}$ can be composed of two terms: spring elastic stiffness $K_{1}$ and viscoelastic origin stiffness $K_{v e}$ (equations 1, 5 and 6.). The comparison between the foam force response in various strain rates (Figure 10.) shows that the unload phase is the same for all strain rates. So, it seems possible to extract the spring elastic stiffness information about it. This idea opens the way for future research work on how to improve the macroscopic integer model.

Note that, in this paper, $\mathrm{M}$ is considered to be equal to eight for future comparison with fractional model. 
(a) strain rate $=1.0610^{-2} \mathrm{sec}^{-1}$

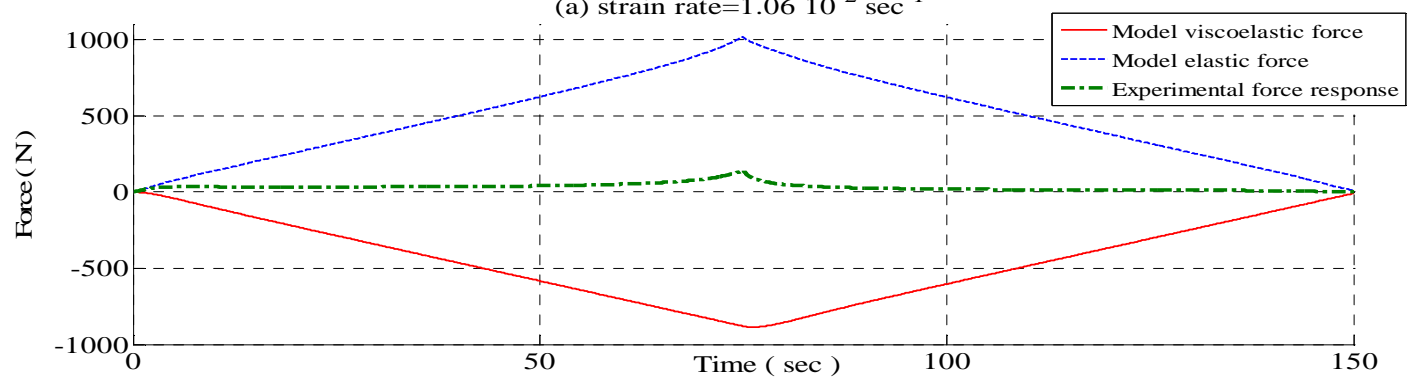

(b) strain rate $=5.310^{-3} \mathrm{sec}^{-1}$

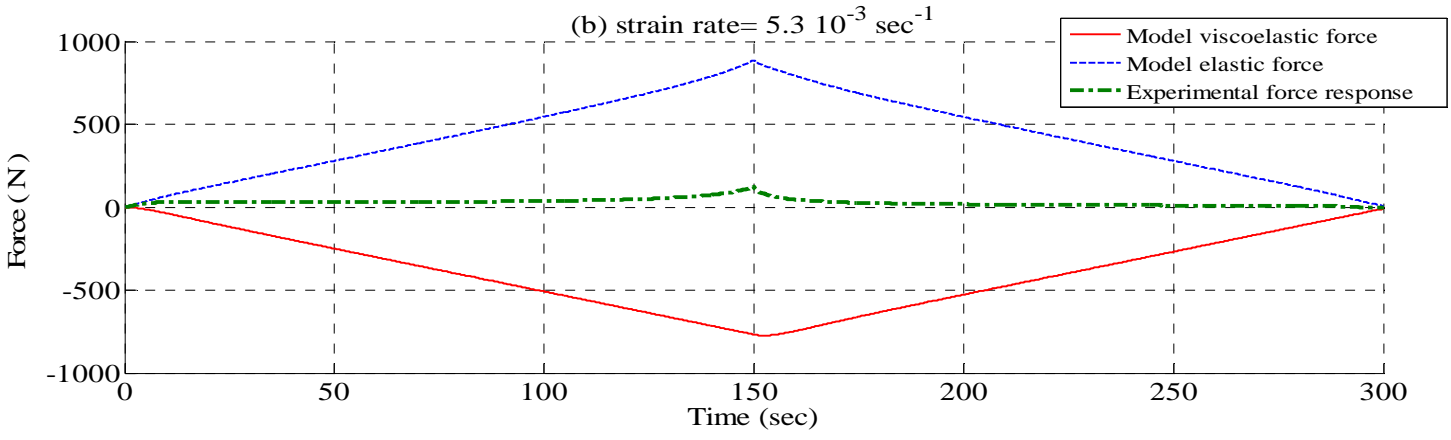

(c) strain rate $=6.6610^{-4} \mathrm{sec}^{-1}$

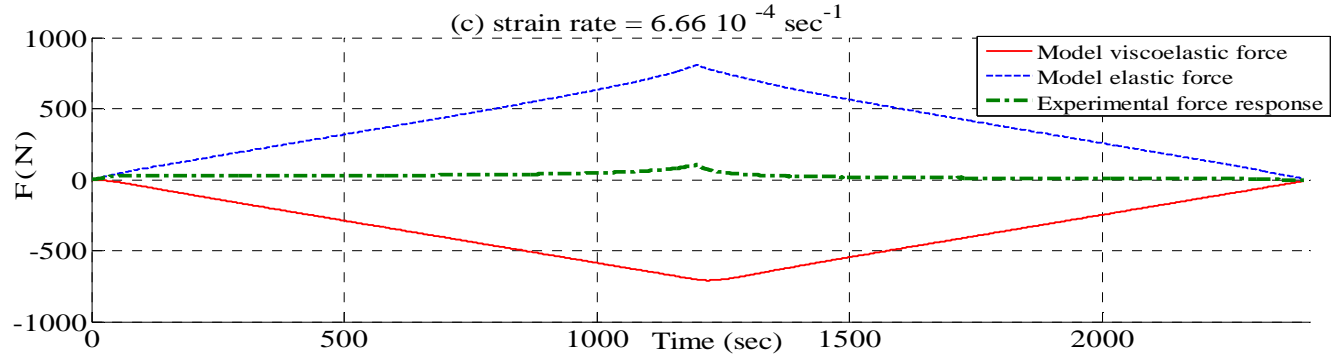

Figure 9. Elastic and viscoelastic forces of foam Type A

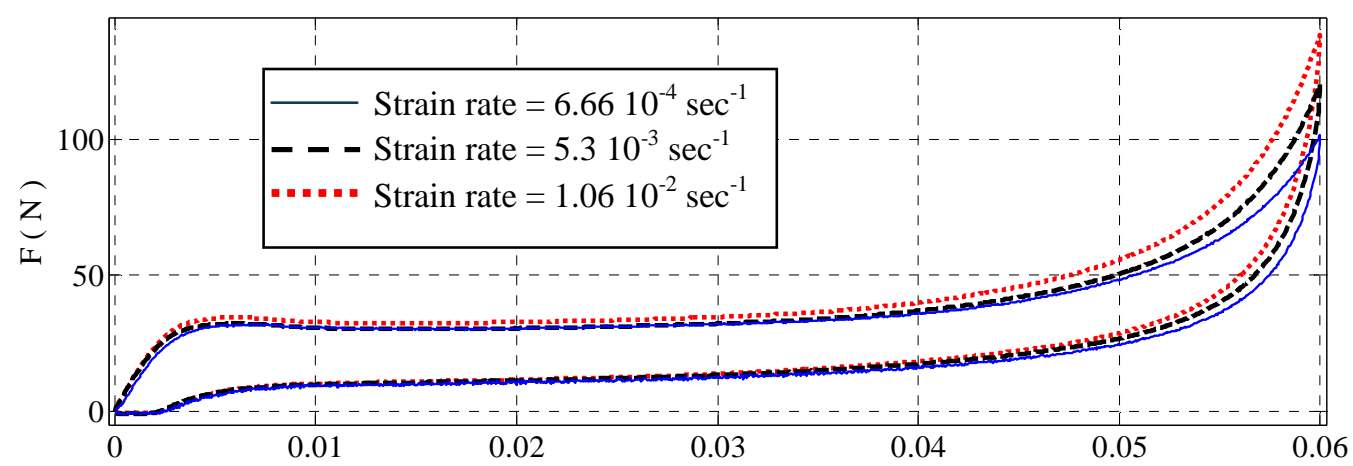

Figure 10. Force response of foam in various strain rates

\section{CONCLUSION}

The difference- forces method is based on the elastic symmetry between the load and unload phases. It is able to identify the viscoelastic and global elastic parameters of the macroscopic integer model. The parameter results verify the thermodynamic conditions and symmetry assumption.

The dimensionless viscoelastic parameters are invariant with test conditions. So, it characterizes the foam material. However, the integer model cannot describe the response of nonlinear spring. 


\section{REFERENCES}

1. Gibson, L.J. and Ashby, M.F. (1997). Cellular Solids: Structure and Properties, 2nd edn, Cambridge University Press, Cambridge (UK).

2. Plastics (II): ASTM D3574-05 Standard Test Methods for Flexible Cellular Materials and Molded Urethane Foams, American Society for Testing Material, juin 2010.

3. Goangseup, Zi., Byeong, M.K., Yoon, K.H. and Young H.L. (2008). An experimental study on static behaviour of a GFRP bridge deck filled with a polyurethane foam, Composite Structures, 82(2): 257-268.

4. Tu, Z.H., Shim, V.P.W. and Lim, C.T. (2001). Plastic deformation modes in rigid polyurethane foam under static loading, International Journal of Solids and Structures, 38(50-51): 9267-9279.

5. Deng, R., Davies, P. and Bajaj A.K. (2006). A nonlinear fractional derivative model for large uni-axial deformation behaviour of polyurethane foam, Signal Processing, 86(10): 2728-2743.

6. Dupuis, L.R. and Aubry, E. (2008). Development and comparison of foam comprehensive law in great deformation, In: S.E.M.XI International Congers Orlando, Florida, USA.

7. Njeugna, N., Schacher, L., Adolphe, C.D., Dupuis, L.R. and Aubry, E. (2008). Comparison of compression behaviour of PU foam and 3D nonwoven, In: Fiber Society 2008 Fall Annual Meeting and Technical Conference, Boucherville, Canada.

8. Ouellet, S., Cronin, D. and Worswick, M. (2006). Compressive response of polymeric foams under quasi-static, medium and high strain rate conditions, Polymer Testing, 25(6):731-743.

9. Ippili, R. K., Davies, P., Bajaj, A.K. and Hagenmeyer, L. (2008). Nonlinear multibody dynamic modelling of seat-occupant system with polyurethane seat and H-point prediction, International Journal of Industrial Ergonomics, 38(5-6):368-383.

10. Deng, R. (2004). Ph.D. Thesis: Modeling and Characterization of flexible polyurethane foam, School of Mechanical Engineering, Purdue University, Indiana USA.

11. Singh, R., Davies, P. and Bajaj, A.K. (2001). Initial condition response of a viscoelastic dynamical system in the presence of dry friction and identification of system parameters, Journal of Sound and Vibration, 239(5):1086-1095.

12. Singh, R., Davies, P. and Bajaj, A.K. (2003). Estimation of the dynamical properties of polyurethane foam through use of Prony series, Journal of Sound and Vibration, 264(5):1005-1043.

13. Singh, R., Davies, P. and Bajaj, A.K. (2003). Identification of Nonlinear and Viscoelastic Properties of Flexible Polyurethane Foam, Nonlinear Dynamics, 34(3):319-346.

14. White, S.W. (1998). Ph.D. Thesis: Dynamic modelling and mesurement of occupied car seats and seating foam, School of Mechanical Engineering, Purdue University, Indiana USA.

15. Bezazi, A. and Scarpa, F. (2009). Tensile fatigue of conventional and negative Poisson's ratio open cell PU foams, International Journal of Fatigue, 31(3): 488-494.

16. Rizov, V. and Mladensky, A. (2009). ModeI fatigue fracture behaviour of Divinucell H-30 structural foam - A non-linear approach, Computational Materials Science, 46(1):255-260.

17. Warren, W.E. and Kraynik, A.M. (1988). The linear elastic propreties of open-cell foams, Journal of Applied Mechanics, 55:341-346.

18. Warren, W.E. and Kraynik, A.M. (1991). The nonlinear elastic behaviour of open-cell foam, Journal of Applied Mechanics, 58:376-381. 
19. Song, Y., Wang, Z., Zhao, L. and Luo, J. (2010). Dynamic crushing behaviour of 3D closed-cell foams based on Voronoi random model, Materials \& Design, 31(9):42814289.

20. Zhu, H.X., Thorpe, S.M. and Windle A. H. (2006). The effect of cell irregularity on the high strain compression of 2D Voronoi honeycombs, International Journal of Solids and Structures, 43(5):1061-1078.

21. Yeoh, O.H. (1993). Some Forms of the Strain Energy Function for Rubber, Rubber Chemistry and Technology, 66:754-771.

22. Arruda, E.M. and Boyce, M.C. (1993). A Three-Dimensional Constitutive Model for the Large Stretch Behaviour of Rubber Elastic Materials, Journal of the Mechanics and Physics of Solids, 41(2):389-412.

23. Treloar, L.R.G. (1975). The Physics of Rubber Elasticity, 3nd end, Clarendon Press, Oxford.

24. Storåkers, B. (1986). On Material Representation and Constitutive Branching in Finite Compressible Elasticity, Journal of the Mechanics and Physics of Solids, 34(2):125145.

25. Ogden, R.W. (1972). Large deformation istropic elasticity: on the correlation of theory and experiment for compressible rubber like solids, Proceedings of the Royal Society of London. Series A, Mathematical and Physical Sciences, 328 (1575):567-583.. 